\title{
Préface de la première édition
}

La naissance de la physique quantique date d'un siècle et cette description des phénomènes physiques, qui a transformé notre vision du monde, n'est toujours pas remise en cause, ce qui est exceptionnel pour une théorie scientifique. Ses prédictions ont toujours été vérifiées par l'expérience avec une précision impressionnante. Les concepts fondamentaux, comme les amplitudes de probabilité, les superpositions linéaires d'états, qui semblent si étranges pour notre intuition quand on les rencontre pour la première fois, restent toujours essentiels. Une évolution importante s'est toutefois manifestée au cours des dernières décennies. Les progrès spectaculaires des techniques d'observation, des méthodes de manipulation des atomes, permettent maintenant de réaliser des expériences si délicates qu'elle n'étaient considérées que comme des "expériences de pensée" par les pères fondateurs de la mécanique quantique. L'existence des corrélations quantiques "non séparables", qui est à la base du "paradoxe" de Einstein-Podolsky-Rosen et qui violent les fameuses inégalités de Bell, a pu être confirmée expérimentalement avec une grande précision. Les états "intriqués" de deux systèmes, qui manifestent de telles corrélations quantiques, sont mieux compris, et même utilisés pour des applications concrètes, comme la cryptographie quantique. L'intrication d'un appareil de mesure avec son environnement se révèle une piste intéressante pour une meilleure compréhension du processus de mesure.

Parallèlement à ces progrès conceptuels, on assiste également à une invasion de notre monde quotidien par des dispositifs dont le principe de fonctionnement repose sur des phénomènes quantiques. Les sources laser qui sont utilisées pour la lecture des disques compacts, l'ophtalmologie ou les télécommunications optiques, sont basées sur l'amplification de la lumière par des systèmes atomiques dont les populations sont inversées. La résonance magnétique des noyaux des atomes est couramment utilisée dans les hôpitaux pour prendre des images de plus en plus précises des organes du corps humain. Des millions de transistors sont inclus dans les puces qui permettent à nos ordinateurs d'effectuer des opérations à des vitesses prodigieuses. 
Il est donc clair qu'un enseignement moderne de la physique quantique doit tenir compte de ces développements, pour donner à l'étudiant ou au chercheur qui désire s'instruire une image plus précise des progrès réalisés et pour accroître sa motivation de mieux comprendre les phénomènes physiques dont l'importance conceptuelle et pratique est de plus en plus évidente. C'est ce défi qu'essaie de relever avec succès Michel Le Bellac dans le présent ouvrage.

Chacun des 14 chapitres de ce livre contient en effet, en plus d'un exposé clair et concis des notions de base, de nombreuses discussions présentant des développements conceptuels ou expérimentaux récents, qui permettent au lecteur de se faire une idée précise des avancées de la discipline et de ses grandes tendances d'évolution. Le chapitre 6 sur les états intriqués est bien caractéristique d'un tel choix de présentation. Au lieu de mettre l'accent sur les propriétés mathématiques du produit tensoriel de deux espaces d'états, ce qui est un peu austère et rébarbatif, ce chapitre préfère centrer la discussion sur la notion d'intrication, et introduire de nombreux exemples de développements théoriques et expérimentaux (dont certains sont très récents) : inégalités de Bell, tests de ces inégalités, en particulier les plus récents utilisant la conversion paramétrique, les états GHZ (Greenberger, Horne, Zeilinger), la notion de décohérence illustrée par des expériences modernes d'électrodynamique en cavité, et qui sera reprise plus en détail dans une annexe, la téléportation. Comme on le voit, il est difficile d'imaginer une immersion plus complète dans l'un des domaines les plus actifs actuellement de la physique quantique. De nombreux exemples de présentation moderne peuvent être donnés à propos d'autres chapitres : interférences d'ondes de de Broglie réalisées avec des neutrons lents, ou des atomes refroidis par laser ; microscopie à effet tunnel ; fluctuations du champ quantique et effet Casimir ; transformations de jauge non abéliennes; équations de Bloch optiques; forces radiatives exercées par des faisceaux laser sur des atomes; piège magnéto-optique; oscillations de Rabi dans le vide d'une cavité, etc.

Je suis vraiment admiratif devant l'effort fait par l'auteur pour donner à son lecteur une vision si moderne et si attrayante de la physique quantique. Certes, les développements décrits ne peuvent pas toujours être analysés en grand détail, et le lecteur devra fournir un effort personnel pour parvenir à une compréhension plus approfondie du sujet étudié. Il sera aidé en cela par la bibliographie détaillée qu'il trouvera, soit au cours du chapitre sous forme de note de bas de page, soit à la fin de chaque chapitre. Je suis convaincu qu'un tel ouvrage permettra une meilleure compréhension de la physique quantique et stimulera un plus grand intérêt pour cette discipline aussi centrale. Je remercie Michel Le Bellac pour cette contribution importante qui va certainement donner une image plus vivante de la physique.

Claude Cohen-Tannoudji 